\title{
On the Movement of Multiple Reaction Zones in Porous Media
}

A distributed parameter model has been developed to describe flow and dissolution of different minerals in porous media. Asymptotic solutions are obtained to give the separation of the different mineral reaction fronts and the time for the reaction front to move through the porous core. The experimental observations agree well with theoretical predictions.

YUSUF HEKIM

and

H. S. FOGLER

Department of Chemical Engineering The University of Michigan Ann Arbor, Michigan 48109

\section{SCOPE}

Porous formations found in nature contain a variety of valuable elements such as uranium, vanadium, and copper and minerals such as carbonates, clays, feldspar and quartz. When reactive fluids are injected into porous media to dissolve these species, one finds their rates of dissolution will be different owing to differences in structure and chemical composition. Consequently, multiple reaction zones will develop as the reactive fluid flows through the porous media. Three areas in which the results of this study have direct applications are in-situ mining of minerals, hydrometallurgy and the acid stimulation of oil and gas reservoirs.

Laboratory studies on mineral extraction and acidization are usually carried out using core flood experiments before application in the field. In a typical experiment, the reactive fluid is passed through a porous sandstone core, while the pressure drop across the core and the effluent concentration are monitored as functions of time. The object of this work was to develop a mathematical model which describes the movement of the multiple mineral reaction zones and the depletion of the reactive fluid in porous cores containing minerals which dissolve at different rates. If the minerals present in a particular sandstone dissolve at approximately the same rate, the lumped parameter model (Lund and Fogler, 1975; Fogler and McCune, 1976) satisfactorily describes the movement of a single reaction front through the core. However, for high temperatures, long cores, or long injection times, the lumped parameter model fails to correctly predict the effluent acid concentration and the movement of multiple reaction zones. $A$ distributed parameter model is necessary to describe flow and reaction in porous media for these cases when the core contains minerals which dissolve at significantly different rates.

\section{CONCLUSIONS AND SIGNIFICANCE}

A distributed parameter model has been developed to describe flow and reaction in porous media when more than one mineral is competing for the reactive fluid. The model predicts the movement of multiple reaction zones through a porous core as well as the concentration of the reactive fluid as a function of time and position. It was found that the reaction zones of the various minerals move through the core at the same speed $\lambda$. For $n$ dissolvable minerals

$$
\lambda=\frac{1}{1+\sum_{j=1}^{n} \frac{1}{A c_{j}}}
$$

The distance by which the mineral reaction zones are separated remains constant as they move through the core. The slower reacting minerals retard the breakthrough of the more reactive mineral zones. Asymptotic solutions were obtained to predict the distance of separation, $\Delta \epsilon$, of the mineral reaction fronts for a typical two-mineral dissolution

$$
\Delta \epsilon=\frac{1}{D a_{2} A c_{2}} \ln \left(2+\frac{A c_{1}}{A c_{2}}\right)
$$

as well as the breakthrough time of different mineral zones. The asymptotic solutions agree well with the full numerical solutions.

The model was used to predict the effluent acid concentration for the sandstone cores undergoing acidization. The lumped parameter model predicts that the effluent acid concentration will equal the entering acid concentration shortly after breakthrough, which is not what is observed experimentally. By considering two minerals dissolving at significantly different rates, the distributed parameter model accurately predicts the effluent acid concentration as a function of time. Experimental results obtained in our laboratory as well as data from other investigators were used to verify the model predictions.
Naturally occurring porous media contain a wide variety of valuable elements such as uranium, vanadium and copper and minerals such as carbonates, clays, feldspars and quartz. The injection of reactive fluids into the porous formation can cause these species to dissolve and go into solution. Since the minerals are different from each other in structure and chemical composition, their rates of dissolution will also be different.

Y. Hekim is now with Michigan Consolidated Gas Co., Detroit, MI.

0001-1541-80-1942-0403-\$01.05. "The American Institute of Chemical Engineers, 1980 .
The reactive fluid flows under a pressure gradient through the formation, and the mineral species present are consumed in the process. The percentage of the reactant consumed by each mineral species is, in turn, proportional to the dissolution rate of the mineral. The dissolvable minerals are imbedded in the porous formation in a less soluble quartz matrix. The quartz will remain intact even after the other minerals have been removed. However, the removal of the more soluble minerals is affected by their relative dissolution rates. These differences in rates of reaction will produce multiple reaction zones as the injected 
fluid passes through the formation. The mineral reaction zone is the region where the unreacted acid contacts the unconsumed minerals. These reaction zones may be distinguishable from one mineral to another if the specific reaction rates are significantly different.

The understanding of the movement of these reaction zones is relevant in the study of a vast majority of porous media reactions. Three areas in which the results of this study have direct application are the in-situ mining of minerals, hydrometallurgy and the acid stimulation of oil and natural gas reservoirs. The problem formulation, the model and resulting solutions are virtually identical for these applications, irrespective of origin.

The solution mining of uranium, vanadium and other rare elements from sandstones can be as advantageous in comparison to other existing techniques owing to the low investment cost and to minimal environmental impact associated with it. During the acid leaching of uranium oxides from sedimentary rocks with sulfuric acid, the dissolution of the ore will take place simultaneously with those of carbonates, sulfides and free iron (Merritt, 1971). The quartz is virtually unreactive with the acid and therefore can be considered as inert. Oxidants such as manganese dioxide and sodium chlorate are added to the acid to promote the dissolution. However, since reducing constituents such as sulfides and iron will preferentially consume the oxidant before the uranium, they should be added to the reactive fluid after these reducing species have been removed. This strategy requires the basic ability to predict the movement of the reaction zones in the ore body.

The reactive fluid used in the extraction of uranium in limestone formations consists of a mixture of $\mathrm{Na}_{2} \mathrm{CO}_{3}$ and $\mathrm{NaHCO}_{3}$. This extraction technique is popular with carbonate formations, where the presence of the bicarbonate ion in the reactive fluid is essential in order to form complexes with the uranyl ion and keep it in solution. However, the formation will generate or consume the bicarbonate ion depending on the relative availability of the minerals. In order to keep the uranyl ion in solution, the movement of the bicarbonate reaction zone in relation to the uranium reaction zone needs to be known in order to insure a successful leaching operation.

Two thirds of the world's oil and natural gas are found in sandstone formations. A typical sandstone consists of a quartz matrix, with feldspars and clays providing the bonding between the grains. These minerals, especially the clays, can migrate during the production of the oil and during water injection in secondary oil recovery. As a consequence, one can observe: a large reduction of the permeability in the vicinity of the well bore owing to the accumulation of the clays. A commonly used technique to restore the productivity of the well is the injection of hydrogen fluoride-hydrochloric acid mixtures into the formation. Hydrofluoric acid is the primary reactant which dissolves the minerals, whereas hydrochloric acid is added to prevent the precipitation of the reaction products and to catalyze the reaction between these minerals and hydrogen fluoride. Experiments carried out in our laboratory have shown that feldspars and clays are more reactive than quartz (Fogler, Lund and McCune, 1975). Clays and feldspars are also different in chemical composition and more importantly in structure and may also dissolve at different rates. Consequently, it is necessary to understand the behavior of the mineral reaction zones in order to design effective acid stimulations.

In summary, during the dissolution of the wide variety of minerals by flowing reactive fluids through the pores of sandstone and limestone formations, it is likely to witness the occurrence of distinct reaction zones with varying separations and sharpness. Consequently, it is of interest to determine the favorable conditions under which these reaction zones will be separated and to estimate the displacement between separable zones. When all the minerals being dissolved from the porous medium react with the fluid at virtually the same rate, they can be lumped together and can be considered as one mineral. In this case, the lumped parameter model (Lund and Fogler, 1976; Fogler and McCune, 1976) predicts the flow, reaction and movement of the reaction and permeability fronts in porous media quite well. However, when the minerals dissolve at significantly different rates, the acid is flowing through long cores or the reaction is carried out under conditions where quartz can no longer be considered inert (for example, high temperatures); the lumped parameter model fails to adequately describe the dissolution process. Even for relatively short cores, the model does not satisfactorily describe the effluent acid concentration after long acid injection times. However, the distributed parameter model developed here is able to overcome the deficiencies of the lumped parameter model and satisfactorily describe the dissolution process.

\section{THE MULTIPLE REACTION FRONT MODEL}

The dissolution of minerals from a porous matrix by means of a reactive fluid takes place in two consecutive steps. The first steps is the heterogeneous surface reaction of the reactive fluid with the mineral. In the second step, a homogeneous reaction between the product from the first step and the reactive fluid results in the formation of a host of stable complexes. The heterogeneous step of the overall reaction is the rate determining step in the dissolution process.

Laboratory studies on mineral extraction and acidization are usually carried out using core flood experiments. In these experiments, cylindrical porous cores are placed in a Hassler cell, shown in Figure 1, and the reactive fluid flows axially through the core within the cell. The effluent acid concentration and the pressure drop across the core are easily monitored as a function of time. The response characteristics of the core to a wide range of variables such as flow rate, reactant concentration and temperature of dissolution can be readily determined.

Since convective transport conditions prevail in most all core flood experiments, the axial dispersion of the reactive fluid species may be neglected (McElhiney et al., 1979). Under these circumstances, the differential mole balance for each of the reactive species in the fluid phase flowing axially through the core (Figure 1) takes the following form:

$$
\frac{\partial\left(\phi C_{i}\right)}{\partial t}+\frac{\partial\left(V C_{i}\right)}{\partial x}=\left(R_{s}+R_{h}\right)_{i}
$$

The corresponding differential mole balance for the solid mineral species can be expressed as

$$
\frac{\partial\left[(1-\phi) W_{j}\right]}{\partial t}=r_{j}
$$

The pertinent boundary and initial conditions in the axial flow case are expressed as

$$
\begin{aligned}
& C_{i}(x, t)=\left(C_{0}\right)_{i} \quad \text { at } x=0 \\
& W_{j}(x, t)=\left(W_{0}\right)_{j} \quad \text { at }(\phi x / V) \geq t
\end{aligned}
$$

The reaction rate of the mineral $\left(r_{j}\right)$ is related to the reaction rate of the reactant $\left(R_{s}+R_{h}\right)_{i}$ by the stoichiometric matrix

$$
\left(\boldsymbol{R}_{s}+\boldsymbol{R}_{h}\right)_{i}=\sum_{j=1}^{n}\left[\nu_{i j}\right] r_{j}
$$

The values of the stoichiometric coefficient $\nu_{i j}$ have been

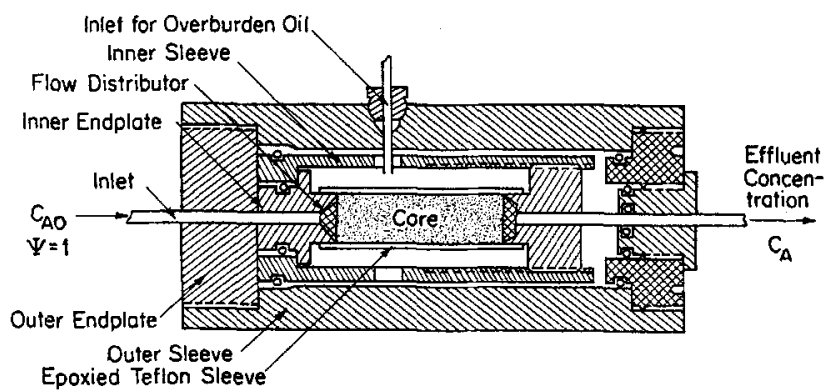

Figure 1. Schematic of diagram of the Hassler cell. 
evaluated from equilibrium relationships for the mud acid dissolution of feldspars and clays as a function of hydrochloric acid concentration, temperature and the extent of mineral dissolved in the reactive fluid (Hekim and Fogler, 1977).

The complete analytical solution for the set of differential mole balances, that is, Equations (1) and (2), is not readily available in closed form, except in the case of lumped parameter model consisting of a single dissolvable mineral and a single reactant (Lund and Fogler, 1976). The lumped parameter model can not predict the observed effluent concentration of the reactive fluid at long injection times. However, for the distributed parameter model described above, in which the minerals dissolve at different rates, one obtains excellent agreement between the predicted and experimental effluent concentrations.

\section{PREDICTION OF THE MOVEMENT OF REACTION FRONTS}

In general, numerical techniques must be used to obtain the solutions to the set of differential mole balances previously derived. However, asymptotic solutions can give significant insight into the understanding of the general trends of the movement of the reaction zones through porous media and reveal characteristics of the behavior of these reaction fronts under different conditions.

By neglecting the change in porosity during dissolution, which is a very realistic assumption (McElhiney, 1979), the differential mole balances can be simplified. In addition, one recognizes the limited accessibility of the mineral species to the reactant owing to dead ended pores, constrictions in the flow channels and to the preferential flow of the fluid through the larger pores (Lund and Fogler, 1976; Swift and Fogler, 1977). Consequently, a certain amount of inaccessible mineral $\left(W_{1}\right)$ exists which should be excluded from the mole balances. Under these circumstances, the reactant and mineral mole balances will be simplified to

$$
\begin{gathered}
\phi_{0} \frac{\partial C_{i}}{\partial t}+V \frac{\partial C_{i}}{\partial x}=\left(R_{s}+R_{h}\right)_{i} \\
\left(1-\phi_{0}\right) \frac{\partial\left[\left(W-W_{1}\right)_{j}\right]}{\partial t}=r_{j}
\end{gathered}
$$

The rate of reaction between the mineral and the reactant can be expressed as a function of the reactant concentration and the accessible mineral content using the rate law (Lund and Fogler, 1976)

$$
-r_{j}=k_{j} C_{i}\left(W-W_{1}\right)_{j}
$$

where the specific reaction rate constant $k_{j}$ is a function of the internal surface area for mineral $j$ in the porous media and the concentration and type of catalytic agents present in the fluid phase. Excellent estimates of $k_{j}$ can be determined from rotating disk experiments coupled with a pore size distribution measurement to determine the macrosurface area (Lund and Fogler, 1976).

When there is only one reactant in the fluid phase causing the mineral dissolution, as is the case in the acid stimulation of oil and gas reservoirs and solution mining, the differential mole balances take the following form:

$$
\begin{array}{r}
\phi_{0} \frac{\partial C}{\partial t}+V \frac{\partial C}{\partial x}=-\left(\sum_{j=1}^{n} \nu_{j} k_{j}\left(W-W_{1}\right)_{j}\right) C \\
\left(1-\phi_{0}\right) \frac{\partial\left(W-W_{1}\right)_{j}}{\partial t}=-k_{j}\left(W-W_{1}\right)_{j} C
\end{array}
$$

These mole balances can be further simplified by utilizing the following dimensionless variables:

$$
\begin{gathered}
\epsilon=\frac{x}{L}, \quad \theta=\frac{t}{\tau}, \quad \tau=\frac{\phi_{0} L}{V} \\
\Psi=\frac{C}{C_{0}}, \quad \eta_{j}=\frac{\left(W-W_{1}\right)_{j}}{\left(W_{0}-W_{1}\right)_{j}}
\end{gathered}
$$

The initial and boundary conditions in dimensionless form are expressed now as:

$$
\begin{array}{ll}
\eta_{j}=1 & \theta \leq \epsilon \\
\psi=1 & \epsilon=0
\end{array}
$$

and the final form of the partial differential equations describing flow and reaction for $n$ dissolving species becomes

$$
\begin{gathered}
\frac{\partial \Psi}{\partial \theta}+\frac{\partial \Psi}{\partial \epsilon}=-\left(\sum_{j=1}^{n} D a_{j} \eta_{j}\right) \Psi \\
\frac{\partial \eta_{j}}{\partial \theta}=-D a_{j} A c_{j} \eta_{j} \Psi \quad(j=1, \ldots, n)
\end{gathered}
$$

where

$$
D a_{j}=\frac{k_{j} \nu_{j}\left(W_{0}-W_{1}\right)_{j} L}{V}
$$

and

$$
A c_{j}=\frac{\phi_{0} C_{0}}{\nu_{j}\left(1-\phi_{0}\right)\left(W_{0}-W_{1}\right)_{j}}
$$

The Damkohler number $\left(D a_{j}\right)$ is the ratio of the rate of consumption of the reactive fluid by mineral $j$ to the rate of convective transport of the reactive fluid. The acid capacity number $\left(A c_{j}\right)$ is the ratio of the fluid reactant available in the pore space to the reactant needed to dissolve all of mineral $j$ accessible per unit volume of porous media.

One of the most desirable pieces of information regarding the behavior of the reaction zones is their rate of propagation through the porous formation and the distance between zones. These reaction zones will behave as shock fronts, that is, steep concentration profiles, if the rate of reaction is many times that of the rate of transport of reactant by convection. Under these conditions, one can obtain asymptotic solutions to predict the rate of movement of these shock fronts (Rhee and Amundson, 1974; Aris and Amundson, 1973).

By choosing an appropriate moving coordinate system, the partial differential equations can be transformed into ordinary differential equations. In case of axial flow through cylindrical cores, one can use the transformation

$$
y=\epsilon-\lambda \theta
$$

which can be derived from the method of characteristics. The necessary and sufficient requirement for the existence of the front is met only at high Damkohler numbers (that is, $D a_{j} \geq 5$ ). The existence of a shock front is equivalent to the existence of a moving coordinate axis $y$ with a speed $\lambda$. If such a shock front exists, then the mineral and reactant concentration profiles will be transformed to

$$
\begin{aligned}
\Psi(\epsilon, \theta) & =\Psi(y) \\
n_{j}(\epsilon, \theta) & =n_{j}(y)
\end{aligned}
$$

Using Equation (11) to transform Equations (9) and (10) into ordinary differential equations gives

$$
\begin{gathered}
(1-\lambda) \frac{d \Psi}{d y}+\left(\sum_{j=1}^{n} D a_{j} n_{j}\right) \Psi=0 \\
\lambda \frac{d \eta_{j}}{d y}=D a_{j} A c_{j} \eta_{j} \Psi
\end{gathered}
$$

Equations (13) and (14) are combined to yield

$$
(1-\lambda) \frac{d \Psi}{d y}+\lambda \sum_{j=1}^{n} \frac{1}{A c_{j}} \frac{d \eta_{j}}{d y}=0
$$

Equation (14) provides the basis for establishing the relationship between the concentrations of any given pair of minerals:

$$
\frac{d \eta_{i}}{d \eta_{j}}=\frac{D a_{i} A c_{i} \eta_{i}}{D a_{j} A c_{j} \eta_{j}}=\frac{P_{i} \eta_{i}}{P_{j} \eta_{j}} \quad(i \neq j)
$$


where $P_{j}$ has been defined as the product of $D a_{j}$ and $A c_{j}$ for a particular mineral species. If a shock front exists, then the solution should satisfy the following boundary conditions:

$$
\begin{aligned}
\frac{d \Psi}{d y}=\frac{d \eta_{j}}{d y}=0 \quad \text { at } y=-\infty \text { and } y=+\infty \\
\eta_{j}=0, \quad \Psi=1 \quad \text { at } y=-\infty \\
\eta_{j}=1, \quad \Psi=0 \quad \text { at } y=+\infty
\end{aligned}
$$

These boundary conditions simply state that the cylindrical core is infinitely long, and the front vanishes at both boundaries. Integrating Equation (16) between the limits of $y=y$ and $y=$ $+\infty$ and rearranging, we get an expression relating the concentration of mineral $j$ to that of mineral $i$ at a fixed position and time:

$$
\eta_{j}=\eta_{i}^{P^{j} / P_{i}}
$$

After we combine Equations (13), (14) and (17), the reactant concentration profile can be obtained from the integration of

$$
(1-\lambda) \frac{d \Psi}{d y}+\lambda \sum_{j=1}^{n} \frac{1}{A c_{j}} \cdot \frac{P_{j}}{P_{i}} \eta_{i}^{\left(P_{j} / P_{i}-1\right)} \frac{d \eta_{i}}{d y}=0
$$

with the limits of integration $y=-\infty$ and $y=y$ to obtain

$$
\Psi=1-\frac{\lambda}{1-\lambda} \sum_{j=1}^{n} \frac{\eta_{i}^{P^{j / P_{i}}}}{A c_{j}}
$$

The mathematical technique used in the evaluation of $\lambda$ has been successfully used for two solute chromatography (Rhee and Amundson, 1974). With this technique, the basic differential mole balance characterizing the system is first differentiated.

$$
\int_{0.5}^{0.5}
$$

The resulting equation can be rearranged and integrated using the boundary conditions formulated above for a shock front. After two integrations, an algebraic equation is obtained which can be solved for $\lambda$, the propagation speed of the shock front. The propagation speed is identical for the fluid and solid reacting species in an infinitely long core.

Differentiating the mineral mole balance, Equation (14), substituting for $d \Psi / d y$ and $\Psi$ from Equations (13) and (18) and integrating between $y=-\infty$ and $y=y$, we get

$$
\begin{aligned}
\frac{\lambda}{P_{i}} \frac{d \eta_{i}}{d y}= & -\frac{\lambda}{1-\lambda} \sum_{j=1}^{n} \frac{P_{j}}{A c_{j}\left(P_{i}+P_{j}\right)} \eta_{i}^{\left(P_{j} / P_{i}+1\right)} \\
& +\eta_{i}-\frac{\lambda}{1-\lambda} \sum_{j=1}^{n} \frac{P_{i}}{A c_{j}\left(P_{i}+P_{j}\right)} \eta_{i}^{\left(P_{j} / P_{i}+1\right)}
\end{aligned}
$$

Use of the boundary condition specified at the limit of $y=\infty$ and simplification gives the propagation speed of the shock front:

$$
\lambda=\frac{1}{1+\sum_{j=1}^{n} \frac{1}{A c_{j}}}
$$

When the reaction fronts of the dissolving minerals are steep relative to the length of the core, then the inverse of the propagation speed will give an excellent approximation of the time necessary for the acid reaction front to reach the end of the core. This time is called the breakthrough time $\theta_{b}$ and is given as

$$
\theta_{b}=\frac{1}{\lambda}=1+\sum_{j=1}^{n} \frac{1}{A c_{j}}
$$

\section{SEPARATION OF THE SHOCK FRONTS AND BREAKTHROUGH TIMES}

For heterogeneous reactions taking place in finite geometries, the shock fronts for the dissolving minerals may become separated from each other if the overall dissolution rates are significantly different. If we assume that the reaction zones have developed into shock fronts (if $D a_{j} \geq 5.0$ ), then the mineral mole balances for each mineral, Equation (14), can be integrated to find the displacement from the midpoint of the preceding front to the midpoint of the succeeding front as shown:

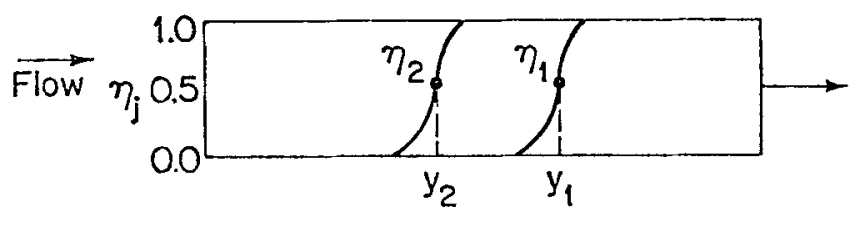

Porous core

The distance between the midpoints is defined as the distance of separation between the mineral fronts. Equations (14) and (18) provide the starting form of the differential equation to be integrated:

$$
\lambda \frac{d \eta_{2}}{d y}=P_{2} \eta_{2}\left[1-\frac{\lambda}{(1-\lambda) A c_{2}} \eta_{2}-\frac{\lambda}{(1-\lambda) A c_{1}} \eta_{2}^{P_{1}{ }^{P_{2}}}\right]
$$

The limits of integration are

$$
\begin{array}{ll}
y=y_{1} & \eta_{1}=0.5 \\
y=y_{2} & \eta_{2}=0.5
\end{array}
$$

If we use Equation (17), the first boundary condition can be written as

$$
\eta_{2}=0.5^{\left(P_{2} / P_{1}\right)} \quad \text { at } y=y_{1}
$$

The equation to be integrated is

$$
\frac{d \eta_{2}}{\eta_{2}\left[1-\frac{\lambda}{(1-\lambda) A c_{2}} \eta_{2}-\frac{\lambda}{(1-\lambda) A c_{1}} \eta_{2}^{P_{1} / P_{2}}\right]}=\int_{y_{1}}^{y_{2}} \frac{P_{2}}{\lambda} d y
$$

The left-hand side of this can not be integrated analytically. However, if the products of the Damkohler and acid capacity numbers are significantly different, that is, $\left(P_{1} / P_{2} \gg 1.0\right.$, then one can neglect the last term in parentheses in the denominator. In addition, the lower limit becomes

$$
(0.5)^{P_{2} / P_{1}} \cong 1.0
$$

Consequently, the integration can easily be carried out to yield the final form:

$$
y_{1}-y_{2}=\frac{\lambda}{P_{2}} \cdot \ln \left(2+\frac{A c_{1}}{A c_{2}}\right)
$$

At any given time $\theta, y_{1}-y_{2}=\epsilon_{1}-\epsilon_{2}$; therefore, the separation between reaction fronts in finite axial flow geometry is estimated for the two mineral system as

$$
\epsilon_{1}-\epsilon_{2}=\frac{\lambda}{P_{2}} \cdot \ln \left(2+\frac{A c_{1}}{A c_{2}}\right)
$$

The propagation speed of the front found for the case of infinitely long flow geometry is valid also for finite geometry. However, owing to the preferential dissolution of the more dissolvable mineral, the less dissolvable minerals will not react until the more reactive mineral species have been consumed. This physical phenomenon is reflected in the separation of the fronts, and consequently the time at which the reaction fronts of individual minerals reach the end of the core (that is, breakthrough) will be different.

The breakthrough time estimated by Equation (21) for finite cores corresponds to the time necessary for the front of the slowest dissolving mineral to reach the end of the core. Using this breakthrough time $\theta_{b}$ as a basis, the breakthrough of the other more reactive mineral fronts are then computed using the distance of separation of the fronts and the propagation speed $\lambda$. For a system which has two dissolvable minerals, the breakthrough time for the second mineral is 


$$
\left(\theta_{b}\right)_{2}=\frac{1}{\lambda}=1+\frac{1}{A c_{1}}+\frac{1}{A c_{2}}
$$

To calculate the breakthrough time for the first mineral, we simply use the shock propagation speed with the distance of separation between shock fronts evaluated in Equation (24):

$$
\left(\theta_{b}\right)_{1}=\frac{1}{\lambda}(1-\Delta \epsilon)=\left(\theta_{b}\right)_{2}-\frac{1}{P_{2}} \cdot \ln \left(2+\frac{A c_{1}}{A c_{2}}\right)
$$

When the number of distinctively dissolving minerals is equal to $n$, where their dissolution rates are sequenced as

$$
P_{i}>P_{i+1}>P_{i+2}>---->P_{n}
$$

then the separation between any of the fronts and the last front can be obtained with the same procedure as outlined for the two mineral case. In closed form, this separation can be formulated as

$$
\begin{aligned}
& \epsilon_{i}-\epsilon_{n}= \sum_{j=i+1}^{n} \frac{\lambda}{P_{j}} \cdot \frac{1}{\left[1-\frac{\lambda}{1-\lambda} \sum_{k=j+1}^{n} \frac{1}{A c_{k}}\right]} \\
& \cdot \ln \frac{\left[2-\frac{\lambda}{(1-\lambda)} \cdot \frac{1}{A c_{j}}-2\left(\frac{\lambda}{1-\lambda} \sum_{k=j+1}^{n} \frac{1}{A c_{k}}\right)\right]}{\left[1-\frac{\lambda}{1-\lambda} \sum_{k=j+1}^{n} \frac{1}{A c_{k}}\right]}
\end{aligned}
$$

If subscript $k$ is greater than the number of minerals reacting, $n$, then $A c_{k}$ is set to infinity ( $1 / A c_{k}$ is zero), and the corresponding terms will drop out from the equation.

\section{NUMERICAL TECHNIQUES}

In the general case, the mole balances need to be solved numerically to evaluate the mineral and reactant concentration profiles as a function of time and position. The asymptotic solutions developed here can only handle the movement of shock fronts were the reaction rate is many times faster than the transport rate.

The numerical technique implemented here has previously been used for fixed-bed adsorption (von Rosenberg, 1969). The technique, which is named as the centered difference technique, substitutes the average of the displacement and time differentials for the partial derivative terms:

$$
\begin{aligned}
& \frac{\partial u}{\partial \theta}=\frac{1}{2}\left(\frac{u_{k, t+1}-u_{k, t}}{\Delta \theta}+\frac{u_{k-1, t+1}-u_{k-1, t}}{\Delta \theta}\right) \\
& \frac{\partial u}{\partial \epsilon}=\frac{1}{2}\left(\frac{u_{k, t+1}-u_{k-1, t+1}}{\Delta \epsilon}+\frac{u_{k, t}-u_{k-1, t}}{\Delta \epsilon}\right)
\end{aligned}
$$

The $k$ 's denote the displacement nodes, and the $t$ 's denote time nodes. At each time step, the mole balances are numerically integrated for the entire length of the core, and the resulting concentration profiles are used for the next computation. The method has proven to be stable and efficient for a wide range of parameter values.

\section{DISCUSSION OF RESULTS}

\section{General Comments}

The movement of reaction fronts through porous media is characterized by expressing the velocity, shape and separation of the mineral and reactant fronts as a function of time. These characteristics are related to the dimensionless parameters $D a_{j}$ and $A c_{j}$. Steep dissolution fronts are characterized by large values of $D a_{j}$, and shallow profiles are characterized by small values of $D a_{j}$. Figure 2 shows the dimensionless reactant fluid and mineral concentration profiles for a typical two dissolvable mineral system with large Damkohler numbers $\left(D a_{j} \geq 10\right)$. One

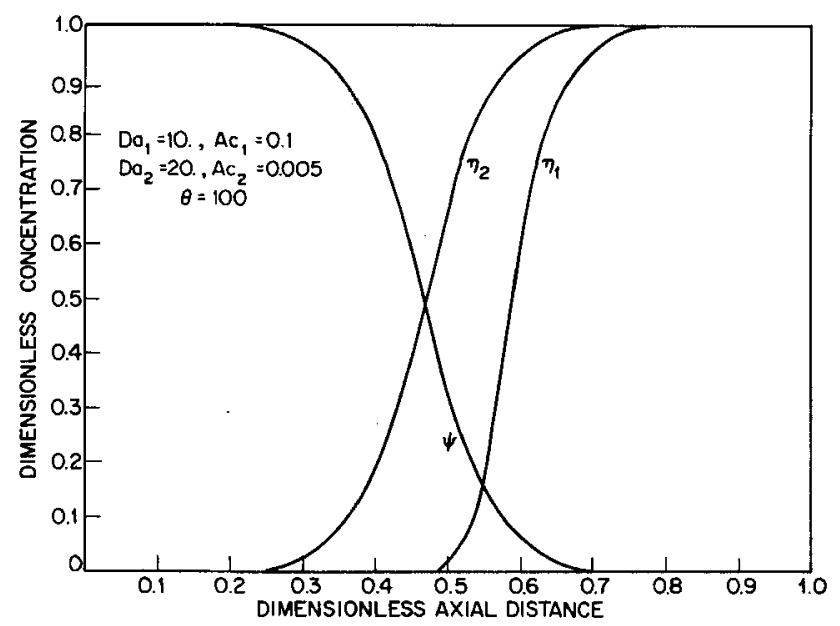

Figure 2. Characteristic mineral and reactant concentration profiles for a two mineral system for two steep fronts.

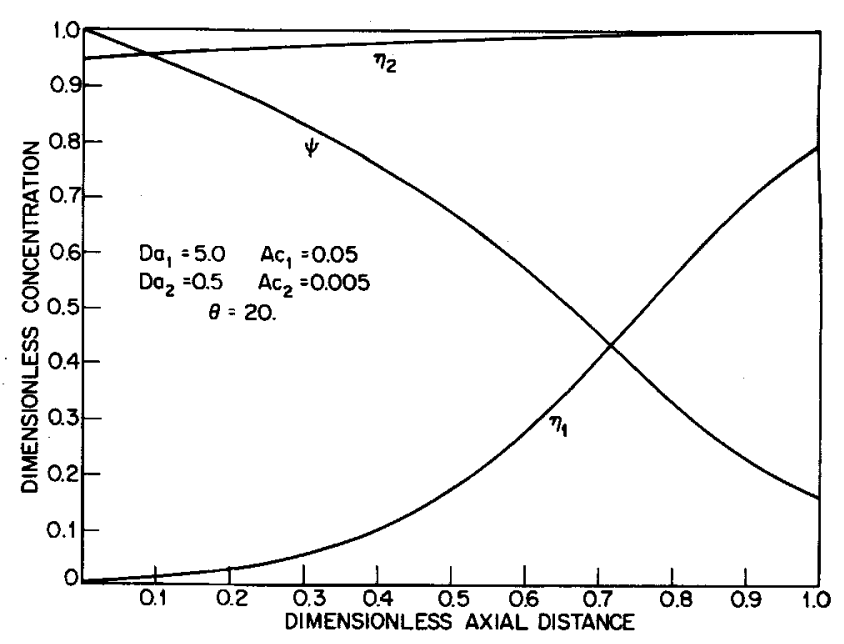

Figure 3. Choracteristic mineral and reactant profiles for a two mineral system for one steep and one shallow front.

observes that the reactant and mineral protiles are relatively steep and well defined in this case.

The product of $D a_{j}$ and $A c_{j}$ defines the relative position of the dissolution front for mineral $j$ with respect to the other mineral fronts. The order of sequence at which the reaction fronts advance through the core is noted in ascending order in terms of the product $D a_{j} \cdot A c_{j}$ for each mineral species. If

$$
(D a \cdot A c)_{A}>(D a \cdot A c)_{B}>(D a \cdot A c)_{C}
$$

then the mineral front for $A$ will move ahead of both of $B$ and $C$, and the mineral front for $B$ will move ahead of $C$. The product of the Damkohler number and acid capacity number expressed in terms of the initially selected variables is given as

$$
P_{j}=D a_{j} \cdot A c_{j}=\frac{\phi_{0} C_{0} k_{j} L}{\left(1-\phi_{0}\right) V}
$$

Since $\phi_{0}, C_{0}, L$, and $V$ are identical for all of the minerals, one can conclude from the fact that if $k_{A}>k_{B}>k_{C}$, then mineral $A$ will be dissolved preferentially in comparison with $B$ and $C$, and $B$ will be dissolved preferentially in comparison with $C$. Individual dissolution fronts become superimposed if the product $D a_{j} \cdot A c_{j}$ is the same.

The reactant and mineral concentration profiles shown in Figure 2 are characteristic of high Damkohler number cases. The other extreme with regard to the shape of the profiles is shown in Figure 3 for a two mineral system, where the Damkohler numbers are small $\left(D a_{j} \leq 5\right)$. The small Damkohler number for mineral $2\left(D a_{j} \leq 1\right)$ is characterized by the almost uniform dissolution through the length of the core. For such 


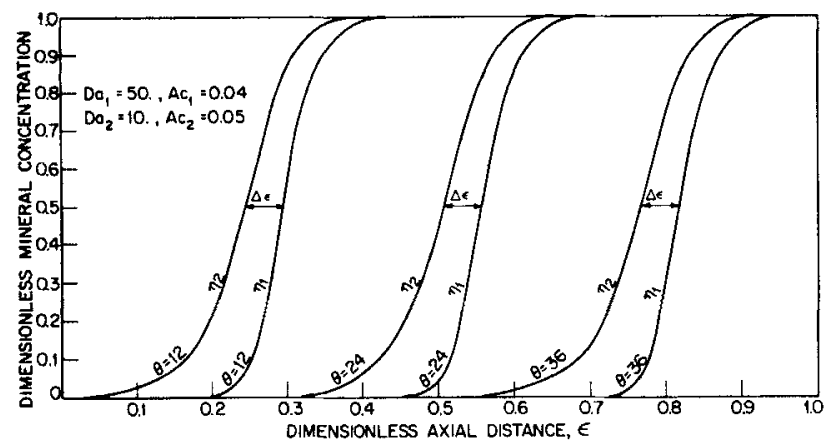

Figure 4. Separation between mineral reaction fronts.

TABLE 1. COMPARISON OF BREAKTHROUGH BY BOTH TECHNIQUES

\begin{tabular}{|c|c|c|c|c|}
\hline \multirow{3}{*}{ Case } & \multicolumn{4}{|c|}{$\begin{array}{l}\text { Breakthrough time } \\
\text { (in pore volumes) }\end{array}$} \\
\hline & $\begin{array}{r}\text { Nur } \\
\text { sol }\end{array}$ & $\begin{array}{l}\text { ical } \\
\text { on }\end{array}$ & $\begin{array}{r}\text { Appr } \\
\text { sol }\end{array}$ & $\begin{array}{l}\text { mate } \\
\text { on }\end{array}$ \\
\hline & $\left(\theta_{b}\right)_{i}$ & $\left(\theta_{b}\right\rangle_{2}$ & $\left(\theta_{b}\right)_{1}$ & $\left(\theta_{b}\right)_{2}$ \\
\hline $\begin{array}{l}P_{1}=2.00 \\
P_{2}=0.05\end{array}$ & 91.2 & 128.8 & 90.2 & 126.0 \\
\hline $\begin{array}{l}P_{1}=2.00 \\
P_{2}=0.05\end{array}$ & 176.1 & 226.5 & 179.9 & 226.0 \\
\hline
\end{tabular}

cases, the reaction zones associated with the minerals will not develop into reaction shock fronts.

The remaining discussion shall be concerned with systems in which only two minerals are dissolving. The more rapidly dissolving minerals will be lumped as feldspars, mineral 1 , and the more slowly dissolving minerals will be lumped as quartz, mineral 2.

For well-developed shock fronts, the numerical solution to the coupled partial differential equations predicts that a finite separation will develop between the mineral fronts as they move through the formation. One observes from the numerical solution for the mineral profiles in Figure 4 that the distance of separation between the dissolution fronts is constant. This distance of separation between the most reactive mineral $i$ and the least dissolvable mineral $n$ can be estimated by the closed form approximation previously derived:

$$
\begin{aligned}
\epsilon_{i}-\epsilon_{n}= & \sum_{j=i+1}^{n} \frac{\lambda}{P_{j}} \cdot \frac{1}{\left[1-\frac{\lambda}{1-\lambda} \sum_{k=j+1}^{n} \frac{1}{A c_{k}}\right]} \\
& \ln \frac{\left[2-\frac{\lambda}{1-\lambda} \frac{1}{A c_{j}}-2\left(\frac{\lambda}{1-\lambda} \sum_{k=j+1}^{n} \frac{1}{A c_{k}}\right)\right]}{\left[1-\frac{\lambda}{1-\lambda} \sum_{k=j+1}^{n} \frac{1}{A c_{k}}\right]}
\end{aligned}
$$

The distance of separation between the profiles for the two mineral system in Figure 4 is equal to 0.051 , and the approximate solution gives a value of 0.045 . The approximation obtained for the separation compares favorably with the numerical solution for a variety of cases as long as the reaction zones have developed into well-defined shock fronts.

In cases where the reaction zones have developed into shock fronts, the breakthrough times for the reacting fluid as well as for each of the dissolvable minerals can be estimated with the aid of the asymptotic solution. Table 1 summarizes the comparison of the breakthrough times estimated with the numerical solution and with the analytical approximation given by Equations (25) and (26). The comparison has been found to be exceptionally good in cases of steep mineral fronts with a finite separation.

The effluent concentration from sandstone cores in acid core flood experiments (Figure 1) can be measured by a number of analytical techniques. The dimensionless effuent concentration

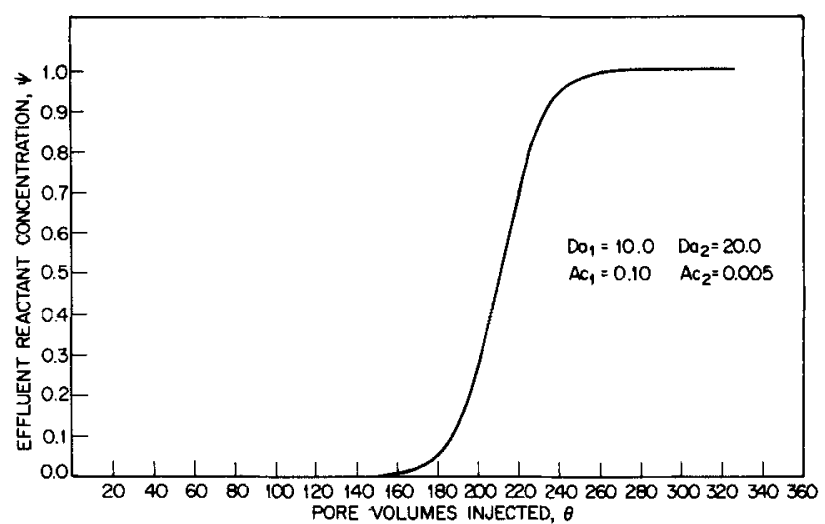

Figure 5. Effluent reactant concentration profiles for the case of high Damkohler numbers.

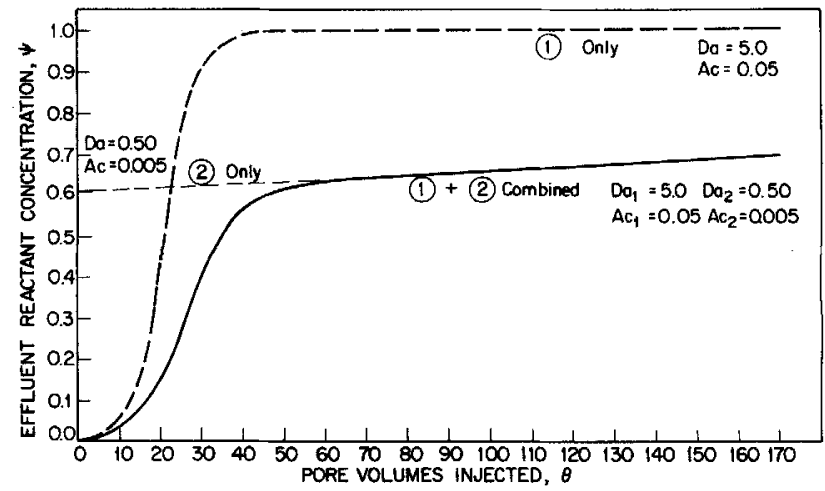

Figure 6. Effluent reactant concentration profiles for the cose of significontly different Damkohler numbers.

for the fluid reactant, that is, acid, has been plotted vs. pore volumes injected in Figures 5 and 6 . Figure 5 is characteristic of the case where both of the minerals present will dissolve at a rate which corresponds to relatively large Damkohler numbers. This is characteristic of porous media reactions, where the reaction rate is many times that of the rate of convective transport of the reactant through the core. Under these circumstances, the dimensionless breakthrough time $\theta_{b}$ is easily determined.

In the lumped parameter model, the dissolvable minerals, lumped as feldspars, are dissolving from an inert quartz matrix. As can be seen from the dashed line numbered 1 in Figure 6, this model predicts the effluent acid concentration to be equal to the inlet concentration shortly after breakthrough. However, most experimental observations show that the effluent acid concentration reaches only 60 to $90 \%$ of the entering acid concentration, even after long injection times. Consequently, while the lumped parameter model successfully predicts the breakthrough time, it does not adequately describe the effluent acid concentration for long cores, high temperatures, or long injection times. The dashed line numbered 2 in Figure 6 is predicted from the lumped parameter model for the case of low Damkohler numbers. This line is representative of minerals with low specific reaction rates or with reactants which are injected at high flow rates. While this case predicts the correct effluent acid profile, it does not predict the observed breakthrough. However, the distributed parameter model with two dissolving minerals, that is, quartz and feldspars, can predict both the breakthrough of the rapidly dissolving mineral and the effluent concentration as a function of time as shown by the solid line in Figure 6. This solid curve is characteristic of two mineral systems, with one of the minerals having a large Damkohler number $\left(D a_{j}=5\right.$.) and the other with a Damkohler number which is more than an order of magnitude smaller.

\section{Comparison of Experimental Observations and Theoretical Predictions}

The lumped parameter model gives the dimensionless 
effluent concentration in terms of the Damkohler and acid capacity numbers and the pore volumes of reactant injected (Lund and Fogler, 1976) as

$$
\Psi=\frac{1}{1+\exp \left[D a A c\left(1+\frac{1}{A c}\right)-D a A c \theta\right]-\exp [D a A c(1-\theta)]}
$$

Rearrangement of Equation (31) results in

$$
\begin{aligned}
& \ln \left(\frac{1}{\Psi}-1\right)=-D a A c \theta \\
&+\ln \left[\exp \left[D a A c\left(1+\frac{1}{A c}\right)\right]-\exp (D a A c)\right]
\end{aligned}
$$

If $\ln (1 / \Psi-1)$ is plotted as a function of pore volumes injected, $\theta$, (instead of $\Psi$ vs. $\theta$ ), a clearer picture of the number of ininerals dissolving at different rates and of the breakthrough time is obtained. If the plot is just a single straight line, one can describe the dissolution using the lumped parameter model. The slope of such a line will give the product of the Damkohler and acid capacity numbers. However, if the plot shows two straight line segments of different slopes, then the two minerals are dissolving at significantly different rates.

The data for the stimulation of Berea cores (Hill et al., 1977) with an acid mixture of $1.5 \mathrm{kmole} / \mathrm{m}^{3} \mathrm{HF}-3.3 \mathrm{kmole} / \mathrm{m}^{3}$ hydrochloric acid at $25^{\circ}$ and $52^{\circ} \mathrm{C}$ have been replotted as $\ln (1 / \Psi-1)$ vs. $\theta$ in Figures 7 and 8 . These data were obtained in a system similar to that shown in Figure 1. In both cases, two distinctive linear segments are obtained. The shallower portion is attributed to the dissolution of quartz, the major but the slowest dissolving constituent of the sandstone. The steeper portion corresponds to the dissolution of the more rapidly dissolving minerals, such as clays and feldspars. The two segments join together as a smooth curve, indicating the transition region where the dissolution rates become comparable. The intersection of the extrapolations of these two linear segments provides a more definitive breakthrough time for the more readily dissolvable mineral when more than one mineral is being consumed in the core. The number of pore volumes or dimensionless breakthrough time is the time necessary for the mineral reaction zone to move through the core. From Figure 8 , one observes a breakthrough time of 38 pore volumes at $52^{\circ} \mathrm{C}$.

Since quartz is the slowest reacting mineral in sandstones, the dissolution of the rest of minerals lumped as feldspars will be completed after breakthrough has been achieved, and quartz alone will continue to dissolve, therefore, the dissolution of quartz can be expressed using the lumped parameter model after the rest of the accessible minerals have been dissolved. The fact that the dissolution of the slowest reacting mineral will affect the breakthrough for the faster dissolving species necessitates the evaluation of the parameters which characterize the dissolution of quartz. Previous studies conducted on the acidization of Phacoides sandstone have provided the parameters which are

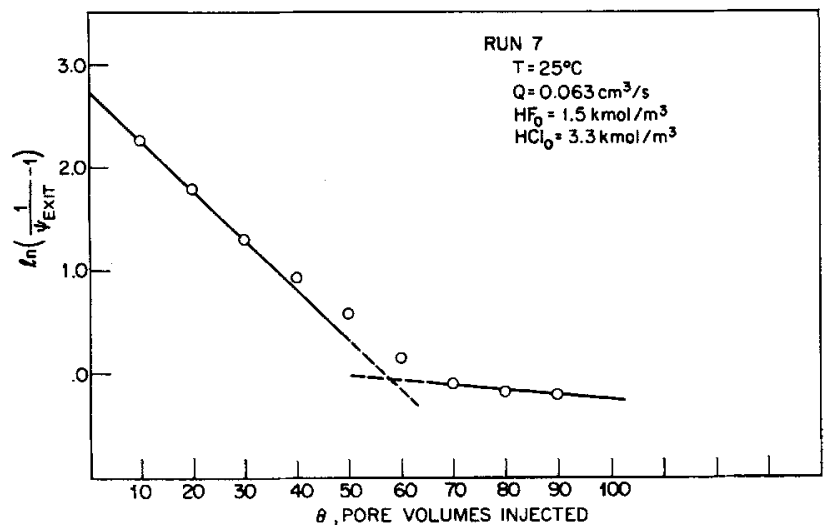

Figure 7. Effluent acid concentration as a function of pore volumes injected for Berea sondstone ot elevated temperature (Hill et al., 1977). characteristic to the dissolution of the class of minerals grouped as the feldspars (Lund, 1974).

Data reported by Hill et al. (1977) on the acidization of Berea

sandstone will be used to evaluate the dissolution parameters for quartz and to make comparisons with theoretical predictions. Figures 7 and 8 have been chosen as the basis for estimating the Damkohler number and acid capacity number for quartz at $25^{\circ}$ and $52^{\circ} \mathrm{C}$, respectively. These experiments were chosen because they correspond to the lowest injection rates, consequently the steepest reaction fronts. At $25^{\circ} \mathrm{C}$, the slope of the shallow segment in Figure 7 gives the product $(-D a \cdot A C)$ for quartz. The extrapolated intercept of this segment is equal to $\ln [\exp (D a A c+D a)-\exp (D a A c)]$. The Damkohler number can be evaluated from the intercept and the acid capacity number then estimated from the product $D a A c$. The same procedure is used to evaluate the Damkohler and acid capacity numbers at $52^{\circ} \mathrm{C}$. These parameters for quartz are best reported in terms of the groups $D a / \tau$ and $A c \nu / C_{0}$ which are truly characteristic of the porous formation to be acidized, which in this case is Berea sandstone.

$$
\begin{array}{ccc}
\mathrm{Temp}\left({ }^{\circ} \mathrm{C}\right) & D a / \tau\left(\mathrm{s}^{-1}\right) \times 10^{3} & A c \nu / C_{0}\left(\mathrm{~m}^{3} / \mathrm{mole}\right) \times 10^{5} \\
25 & 4.2 & 1.47 \\
52 & 11.5 & 1.49
\end{array}
$$

Since the product $(D a A c)$ is proportional to the specific reaction constant, this information can be used to determine the activation energy for the dissolution of quartz. The activation energy in the range of $25^{\circ}$ to $52^{\circ} \mathrm{C}$ is evaluated to be $29.9 \mathrm{~kJ} / \mathrm{mole}$ which agrees well with $30.6 \mathrm{~kJ} / \mathrm{mole}$ from kinetic rate data reported by Lund (1974).

The acid capacity number reflects the fraction of the dissolving mineral to which the reactant has access for reaction. The acid capacity number for quartz gives the fraction of accessible mineral at 0.42 to 0.45 . This fraction is comparable to that found from the acid capacity and aluminum petrologs of acidized cores for feldspar dissolution in Phacoides sandstone (Lund and Fogler, 1976).

Using these values of $D a / \tau$ and $A c \nu / C_{0}$ for quartz obtained from Hill's data, we should now reanalyze the data for Phacoides sandstone, considering the dissolution of two minerals, feldspars and quartz. For Phacoides sandstone, the dissolution parameters for the feldspar are

$$
\frac{D a}{\tau}=0.133 \mathrm{~s}^{-1}\left(52^{\circ} \mathrm{C}\right) \frac{A c \nu}{C_{0}}=1.4 \times 10^{-4} \mathrm{~m}^{3} / \mathrm{mole}
$$

However, the parameters for quartz, namely, the Damkohler and acid capacity numbers, can be estimated from the respective parameters evaluated from the Berea type of sandstone.

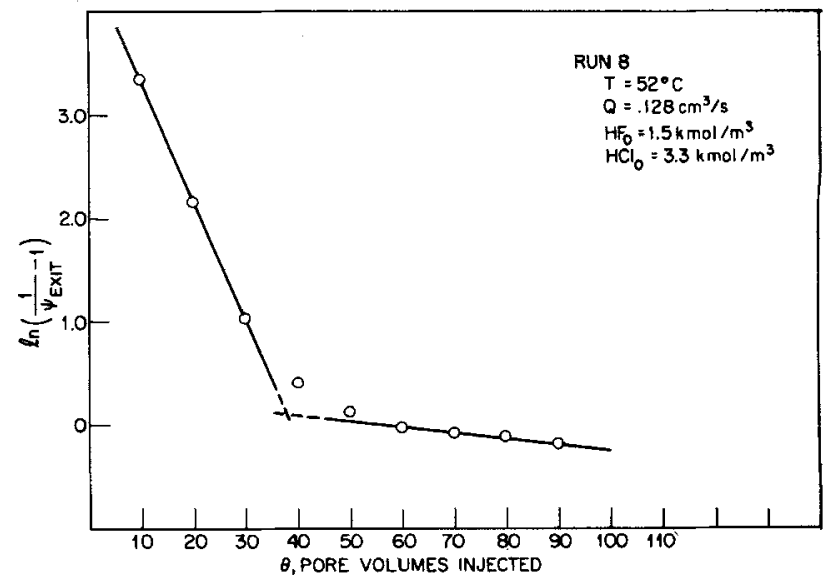

Figure 8. Effluent acid concentration os a function of pore volumes injected for Berea sondstone at ambient temperature (Hill et al., 1977). 


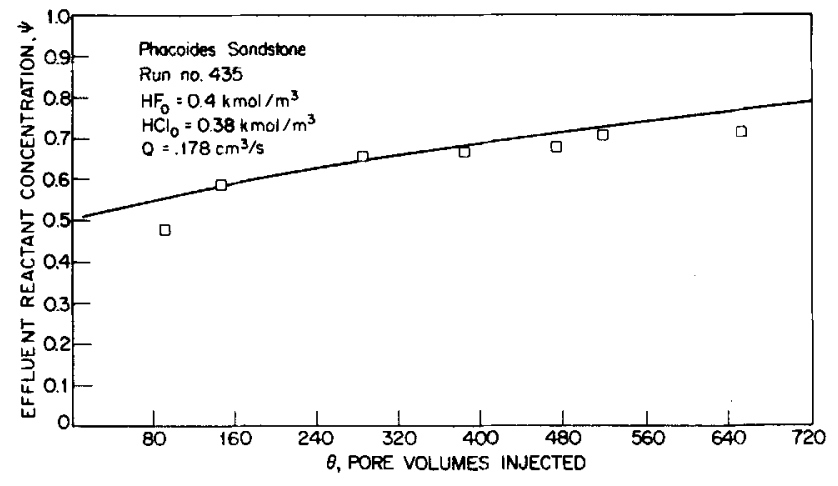

Figure 9. Effluent acid concentration as a function of pore volumes injected for Phocoides sandstone (Lund and Fogler, 1976).

A technique to estimate the parameters $\dot{D a}$ and $A c$ has been successfully used in the application of the lumped parameter model to a wide variety of sandstone cores (Fogler and McCune, 1976). The parameters are estimated from knowledge of the temperature and sandstone porosity and mineralogical composition. The same technique can be utilized to estimate the quartz dissolution parameters for any other type of sandstone. The basic assumption in applying this method of transformation is that the internal surface area is essentially constant from one type of sandstone to another, and the fraction of mineral accessible to the acid is approximately the same; that is, it is within the range of 0.40 to 0.50 for the most common sandstone formations.

Knowing the characteristic groups $(D a / \tau)_{A}$ and $\left(A C \nu / C_{0}\right)_{A}$ for any sandstone $A$, one can calculate these groups for any other sandstone $B$ as follows:

$$
\begin{aligned}
& \left(\frac{D a}{\tau}\right)_{B}=\left(\frac{D a}{\tau}\right)_{A} \cdot \frac{\left(W_{0}\right)_{B}}{\left(W_{0}\right)_{A}} \\
& \cdot \frac{\left(\phi_{0}\right)_{A}}{\left(\phi_{0}\right)_{B}} \cdot \exp \left[-\frac{\Delta E}{R}\left(\frac{1}{T_{B}}-\frac{1}{T_{A}}\right)\right] \\
& \left(\frac{A c \nu}{C_{0}}\right)_{B}=\left(\frac{A c \nu}{C_{0}}\right)_{A} \cdot \frac{\left(W_{0}\right)_{A}}{\left(W_{0}\right)_{B}} \cdot \frac{\left(\phi_{0}\right)_{B}}{\left(\phi_{0}\right)_{A}} \cdot \frac{\left(1-\phi_{0}\right)_{A}}{\left(1-\phi_{0}\right)_{B}}
\end{aligned}
$$

After $D a / \tau$ and $A C \nu / C_{0}$ have been evaluated for the quartz in Phacoides sandstone, the acidization parameters $D a$ and $A c$ can be estimated by substituting the values of $\tau, \nu$ and $C_{0}$ for the specific acidization case. Finally, the set of equations which were derived as mole balances are solved numerically to predict the effluent concentration. The final set of parameters for run 435 are tabulated below:

$$
\begin{array}{ccccc} 
& \multicolumn{2}{c}{\text { Feldspar }} & \multicolumn{2}{c}{\text { Quartz }} \\
\text { Run No. } & D a & A c & D a & A c \\
435 & 0.591 & 0.0037 & 0.084 & 0.0008
\end{array}
$$

The results obtained from the numerical solution are plotted in Figure 9 together with the data to show the exceptional agreement between theory and experiment when the minerals are dissolving at different rates.

Finally, the multiple reaction front model was used to predict the effluent reactant concentration for Hill's experiments on the acidization of Berea sandstone for a variety of experimental conditions. The characteristic groups $A c \nu / C_{0}$ and $D a / \tau$ for quartz have already been determined for this sandstone from Figures 7 and 8 , while these same groups for feldspar were determined using the technique described above. These two sets of parameters were utilized in the numerical solution of Equations (13) and (14) to predict the effluent concentration as a function of pore volumes acid injected. Figures 10 through 11 show the comparison of Hill's data and the theoretical prediction. The dashed line in Figure 10 shows the predicted effluent concentration when variations in porosity are accounted for.

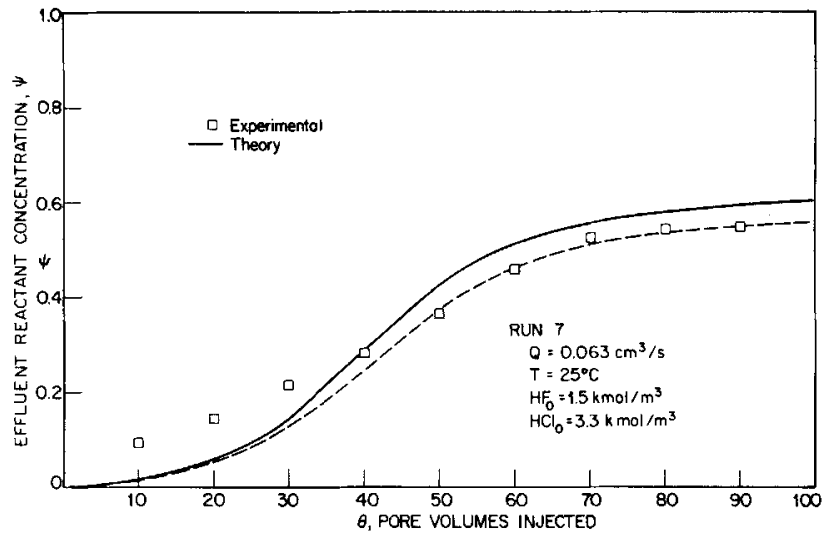

Figure 10. Effluent acid concentration os a function of pore volumes injected for Berea sondstone (Hill et ol., 1977).

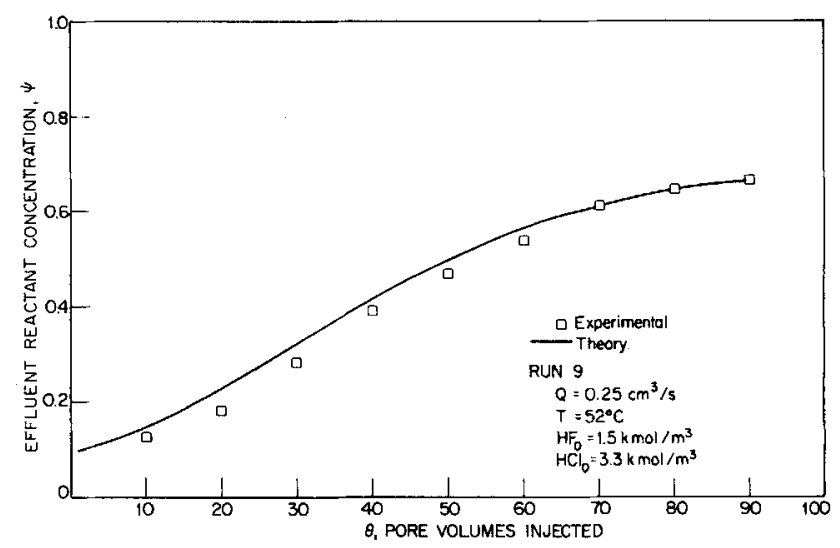

Figure 11. Effluent acid concentration as a function of pore volumes injected for Bereo sandstone (Hill et ol., 1977).

When we consider the range of injection rates and temperatures used, the agreement obtained between data and theory is exceptionally good. The characteristic groups $D a / \tau$ and $A c \nu / C_{0}$ are fixed for each temperature, so that there were no adjustable parameters in the model in arriving at this good agreement. The addition of quartz to the dissolution model clearly shows that the application of the distributed parameter model to these two mineralogically different sandstones is quite successful.

\section{NOTATION}

$A c_{j} \quad=$ acid capacity number for species $j$

$C=$ reactant concentration, $\mathrm{mole} / \mathrm{m}^{3}$

$C_{0} \quad=$ inlet concentration of reactant, mole $/ \mathrm{m}^{3}$

$D a_{j} \quad=$ Damkohler number for species $j$

$k_{j} \quad=$ specific reaction rate for species $j, \mathrm{~m}^{3} \mathrm{~mole} / \mathrm{s}$

$L \quad=$ length of core, $m$

$P_{j} \quad=$ product of Damkohler and acid capacity numbers for mineral $j$

$R=$ generation rate of reactant, $\mathrm{mole} / \mathrm{m}^{3}$ of bed $/ \mathrm{s}$

$r_{j}=$ generation rate of mineral $j$, mole $/ \mathrm{m}^{3}$ of bed $/ \mathrm{s}$

$t \quad=$ time, $\mathrm{s}$

$V \quad=$ superficial velocity of reactant, $\mathrm{m} / \mathrm{s}$

$W=$ mineral concentration, mole $/ \mathrm{m}^{3}$ of solids

$W_{0} \quad=$ initial concentration of mineral, mole $/ \mathrm{m}^{3}$

$W_{1} \quad=$ inaccessible mineral concentration, $\mathrm{mole} / \mathrm{m}^{3}$ of solids

$x=$ axial displacement, $\mathrm{m}$

$y \quad=$ moving coordinate axis

\section{Greek Letters}

$\epsilon=$ dimensionless axial displacement

$\eta \quad=$ dimensionless reactant concentration

$\theta \quad=$ dimensionless time, pore volumes reactant injected

$\lambda=$ propagation speed of shock front 
$\nu \quad=$ stoichiometric coefficient (moles reactant consumed $/$ mole of mineral reacted)

$\tau=$ space time, $s$

$\psi \quad=$ dimensionless reactant concentration

$\phi \quad=$ porosity

\section{Subseripts}

b = breakthrough

$h \quad=$ homogeneous phase reaction

$i \quad=$ reactant species

$j=$ mineral species

$n \quad=$ last of the moving fronts

$s \quad=$ surface reaction
Hekim, Y., and H. S. Fogler, "Acidization VI. On the Equilibrium Relationships and Stoichiometry of Reaction in Mud Acid,"ibid., 32, 1, (1977).

Hill, A. D., D. M. Lindsay and R. S. Schechter, "Sandstone Acidizing: The Development of Design Methods," International Symposium on Oil Field and Geothermal Chemistry, Society of Petroleum Engineers, Paper No. 6607, Dallas, Tex. (1977)

Lund, K., and H. S. Fegler, "Acidization V. On the Prediction of the Movement of Acid and Permeability Fronts in Porous Media," Chem. Eng. Sci., 31, 381 (1976).

Lund, K., Ph.D. thesis, "On the Acidization of Sandstone," The Univ. of Mich., Ann Arbor (1974).

McElhiney, J. E., A. L. Schalge, R. S. McKnight and J. R. Roberson, "Coreflood Acidizing: Model, Measurement and Methodology," International Symposium on Oil Field and Geothermal Chemistry, Society of Petroleum Engineers, Paper No. 7891, Houston, Tex. (1979).

APPENDIX: QUARTZ DISSOLUTION PARAMETERS FROM DATA OF A. D. HILL et al., (1977)

$\begin{array}{ccc}\text { Figure } & T\left({ }^{\circ} \mathrm{C}\right) & D a \cdot A c \\ 7 & 25 & 3.226 \times 10^{-3} \\ 8 & 52 & 4.381 \times 10^{-3}\end{array}$

$\frac{V\left(1-\phi_{0}\right)}{L \phi_{0} C_{0}}$
0.223
0.453

$k_{\text {quartz }}\left(\mathrm{m}^{3} / \mathrm{mole} / \mathrm{s}\right) \times 10^{8}$

1.21

3.31

The activation energy between $25^{\circ}$ and $52^{\circ} \mathrm{C}$ is $29.9 \mathrm{~kJ} / \mathrm{mole}$.

\begin{tabular}{|c|c|c|c|c|c|c|}
\hline Figure & $T\left({ }^{\circ} \mathrm{C}\right)$ & $Q\left(\mathrm{~cm}^{3} / \mathrm{s}\right)$ & $D a_{F}$ & $A c_{F}$ & $D a_{Q}$ & $A c_{Q}$ \\
\hline 10 & 25 & 0.342 & 0.8633 & 0.035 & 0.1139 & 0.0053 \\
\hline 11 & 52 & 0.250 & 1.9495 & 0.037 & 0.4269 & 0.0052 \\
\hline
\end{tabular}

\title{
LITERATURE CITED
}

Aris, R., and N. R. Amundson, Mathematical Modeling in Chemical Engineering, Vol. 2, Prentice Hall, Englewood Cliffs, N. J., (1973).

Carnahan, B., H. A. Luther and J. O. Wilkes, Applied Numerical Methods, Wiley, New York, (1969).

Fogler, H. S., and C. C. McCune, "On the Extension of the Model of Matrix Acid Stimulation to Different Sandstones," AIChE J., 22, 799 (1976).

Fogler, H. S., K. Lund and C. C. McCune, "Acidization III. The Kinetics of the Dissolution of Sodium and Potassium Feldspar in Hydrochloric-Hydrofluoric Acid Mixtures," Chem. Eng. Sci., 30, 825 (1975).

Merrit, R. C., The Extractive Metallurgy of Uranium, Colorado School of Mines Research Institute (1971).

Rhee, H., and N. R. Amundson, "Shock Layer in Two Solute Chromatography: Effect of Axial dispersion and Mass Transfer," Chem. Eng. Sci., 29, 2049, (1974).

Swift, S. T., and H. S. Fogler, "A Note on Capillary Model Developments for Sandstone Acidization," ibid., 32, 399 (1977).

von Rosenherg, D. U., Modern Analytic and Computational Methods for the Numerical Solution of Partial Differential Equations, Elsevier, New York, (1969).

Manuscript received May 10, 1979; revision received October 15, and accepted October 24, 1979.

\section{On the Construction of Dispersion}

\section{Approximations to the Solution of the Convective Diffusion Equation}

\author{
ANTHONY E. DeGANCE \\ Amoco Production Company \\ P.O. Box 591 \\ Tulsa, OK 74102
}

and

We identify dispersion approximations to the transverse average of the solution of the convective diffusion equation on exacting equality of a finite number of axial Hermite moments. The method unifies the dispersion of chemically active and passive solutes and generalizes dispersion theory to arbitrary transverse averages. We emphasize the importance of the scalar results via their application to the dispersion of $\overline{\mathrm{a}}$ system of chemical isomers.

Amoco Production Company
P.O. Box 591
Tulsa, OK 74102
and
LEWIS E. JOHNS
Department of Chemical Engineering
University of Florida
Gainesville, FL 32611

\section{SCOPE}

In the area average and for chemically inactive solute, the dispersion theory of Gill and Sankarasubramanian generates useful approximations to the transverse average of the solution 0001-1541-80-3571-0411-\$01.05. The American Institute of Chemical Engineers, 1980. of the convective diffusion equation. Because technical difficulties limit the implied construction in nonarea averages and for chemically active solute, a reformulation of the conditions which define the dispersion coefficients is outlined. The result 